Proceedings of the 11th Polish-Japanese Joint Seminar on Micro and Nano Analysis, Gniew, September 11-14, 2016

\title{
Influence of Phase Transformations on Incipient Plasticity of Si-Nanospheres
}

\author{
D. CHROBAK $^{a, *}$ AND R. NOWAK ${ }^{b}$ \\ ${ }^{a}$ Institute of Materials Science, University of Silesia in Katowice, 75 Pułku Piechoty 1, 40-500 Chorzów, Poland \\ ${ }^{b}$ School of Chemical Technology, Aalto University, P.O. Box 16100, 00076 Aalto, Finland
}

\begin{abstract}
Our finding of the current spike effect highlighted for the first time in 2009 offers an enhanced understanding of the link between nanoscale mechanical deformation and electrical behavior, and ultimately suggests key advances in unique phase-change applications in future electronics. Certainly, crystal imperfections affect the properties of the nanoparticles themselves, e.g., their biocompatibility and biodegradability. The potential role of dislocations having a profound impact on the use of Si nanoparticles was largely overlooked, since plastic deformation of bulk $\mathrm{Si}$ is dominated by amorphization and phase transformations. Here we show an effect of bulk $\rightarrow$ nanoparticle transition (deconfinement) on incipient plasticity of Si-nanovolume. Our results provide a fresh insight into the dilemma concerning dislocation or phase transformation origin of nanoscale plastic deformation of semiconductor nanoobjects.
\end{abstract}

DOI: $10.12693 /$ APhysPolA.131.1328

PACS/topics: 62.20.--x, 62.50.-p, 64.70.kg

\section{Introduction}

One of the fundamental questions of science and engineering concerns the nature of deformation of solids when stressed by external forces [1]. The onset of plasticity is traditionally understood in terms of dislocation motion. The study of nanoscale deformation when the surface of crystal is subjected to contact stress has proven that initial displacement transient events occurring in metals are the direct result of dislocation nucleation [2]. Our research revealed that this is not always true: instead of dislocation activity, nanoscale deformation may simply be due to phase transformation (semiconductor $\rightarrow$ metal), as predicted for GaAs by our earlier experiments and atomistic calculations [3]. Using a novel conductive nanoindentation technique, which is highly sensitive to structural changes under pressure [4], we discovered the essential link between this electrical phenomenon (current spike) and the mechanical transient (pop-in) exhibited by GaAs exclusively during nanoscale deformation. The results obtained for GaAs and dramatic impact of crystal imperfections on the functional properties of semiconductors have motivated our studying the nanoscale deformation of Si nanoparticles that is addressed in the present paper.

Silicon, one of the most extensively studied advanced technology materials exhibits a complex pattern of plastic deformation combining amorphization [5,6], phase transformations [5-8], and dislocations [7, 8]. Recent nanoscale compression $[9,10]$ and nanoindentation observations [7] demonstrated that deformation of silicon nanovolumes is markedly different from behavior of the

*corresponding author; e-mail: dariusz.chrobak@us.edu.pl bulk material. The enhanced hardness of Si nanospheres is consistent with the known phenomenon of yield stress increase due to the decrease of dimensions of deformed volumes $[9,10]$. However, the reversible plasticity exhibited by the compressed Si nanoparticles $[9,10]$ cannot be justified in the framework of the theories pertinent to the bulk Si surfaces. Gerberich et al. [9, 10] proposed a model of dislocation-driven onset of plasticity accounting for the unusual behavior of Si nanoparticles. Following the latter idea, we provide a detailed atomistic account of stress-driven deformation in Si nanoparticles based on experimental evidence indicating the effect of nanoscale confinement.

\section{Experimental}

In order to model processes initiated in stressed $\mathrm{Si}$ nanospheres we performed molecular dynamics (MD) simulations (LAMMPS simulation code) designed to match experimental conditions of previously reported nanocompression tests $[9,10]$. MD interactions between silicon atoms were modeled using the Stillinger-Weber $(\mathrm{S}-\mathrm{W})$ potential [11] accurately reflecting elastic behavior and generation of lattice defects in silicon [12]. Simulations were performed for the silicon sphere radius $R=10 \mathrm{~nm}$ and compressed between two rigid parallel plates. Deformation history of the Si nanosphere was captured in contact pressure-strain $\left(p_{c}-\varepsilon\right)$ relationships derived from our MD simulations. Loading was conducted via 0.25 Åsymmetrical displacement $(\delta)$ increments applied to both plates. Consequently, the strain parameter is defined as follows: $\varepsilon=\delta / R$. The atomic structure of the deformed nanosphere was relaxed during 20000 time steps ( $\Delta t=2 \mathrm{fs}$ ) for each displacement increment occurring at a temperature of $300 \mathrm{~K}$. The loading of Si nanospheres accomplished along the [001] direction of the crystal was characterized by the mean contact pressure parameter $p_{c}=P / A$, where contact area $A$ is esti- 
mated via determining the number of Si atoms experiencing non-zero interaction with the contacting rigid surface. The atomistic structural changes during MD simulations was visualized utilizing slip-vector analysis [13] with the threshold value of $1 \AA$.

\section{Results and discussion}

Perfectly elastic behavior of Si nanosphere during initial stages of compression along [001] axis (Fig. 1) conforms to classic continuum mechanics expressed by the 13 decades old Hertzian equation [14]. In agreement with the results of MD simulations by Valentini et al. [15], our MD calculated elastic modulus for the nanosphere $\left(E_{[001]}=138 \mathrm{GPa}\right)$ is lower than the $\approx 160 \mathrm{GPa}$ measured for the bulk Si [10]. The reduction of Young's modulus with decreasing particle size is attributed to the high surface-to-volume ratio of nanospheres [15] consistent with the recent results by Mo et al. [16]. Our MD simulations registered contact pressure reaching $\approx 23 \mathrm{GPa}$ (Fig. 1) matching the experimentally observed elevated yield-point stress of the Si nanospheres $[9,10]$. This value is almost twice higher than the hardness of the bulk Si [9], while nearly identical to the magnitude of peak stress reached at the point of elastic-plastic transition in previous simulations [15].

The simulations with the $\mathrm{S}-\mathrm{W}$ potential reveal that the maximum contact pressure is followed by a sudden, dramatic drop in $p_{c}$ at strain $\varepsilon=0.108$ (Fig. 1). Then the contact pressure continues to gradually decrease approaching hardness $(H)$ of bulk Si at $\approx 12 \mathrm{GPa}$ [9]. The apparent decrease of the contact pressure under the maintained constant rate of displacement increase is tantamount to the pop-in observed during nanoindentation experiments [17-19]. Pop-in, alternatively referred to as the yield point, marks the onset of plasticity in defectfree crystals [17-19]. For load controlled experiments, pop-in appears as rapid displacement increase whereas its incidence under displacement control is evident as a load drop [20].

Plastic deformation in the nanosphere $(R=10 \mathrm{~nm})$ starts at strain $\varepsilon=0.108$, corresponding to the popin event. Four pairs of perfect dislocation loops (the Burgers vector value $|0.5[101]|=3.84 \AA$ ) nucleate on the $\{111\}$ planes at the pop-in extending nearly halfway across the particle (Fig. 2). At higher strain $\varepsilon=0.112$, the development of the dislocations' arrangements proceeds and some of dislocations approach the surface of the nanosphere. Interestingly, release of the load applied right after the pop-in causes sudden expansion of the bottom dislocation that reaches the nanosphere surface (formation of the surface step) while the remaining dislocations disappear when unloading is completed (Fig. 2). Our simulations have shown the complex nature of the nanosphere plastic deformation composed of irreversible as well as reversible dislocations' activity.

It is well documented that induced by nanoindentation the nanoscale deformation of bulk $\mathrm{Si}$ is accompanied with the phase transformation from the initial diamond-

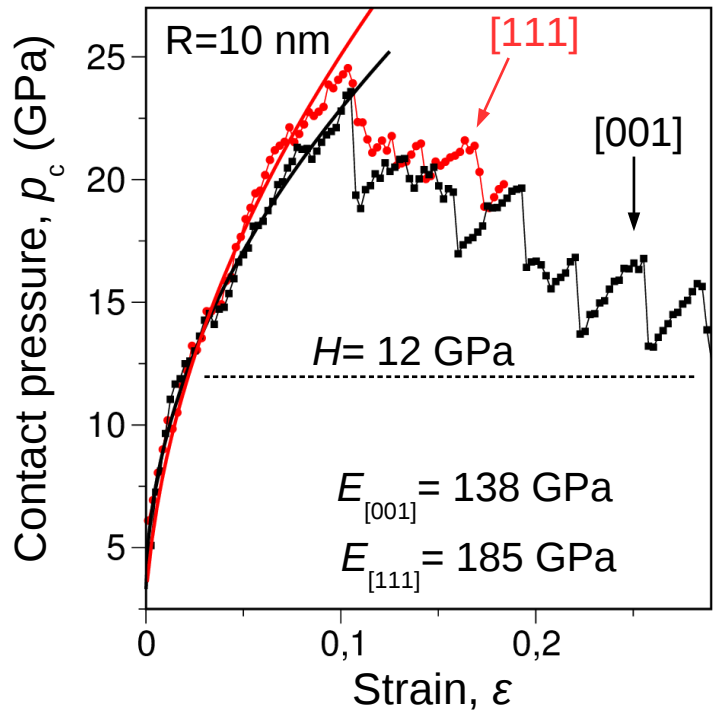

Fig. 1. Results of MD simulations on Si nanosphere of radius $10 \mathrm{~nm}$. The compression axis was selected to be aligned with [001] direction of silicon diamond lattice. The first stage of $p_{c}-\varepsilon$ relationship exhibits Hertzian elastic behavior. Then, at the strain $\varepsilon=0.108$, the plastic deformation starts with the pop-in singularity. Furthermore, the mechanical response of the nanosphere contains the next pop-ins, and the contact pressure $p_{c}$ gradually approaches the value of silicon hardness $(\approx 12 \mathrm{GPa})$. For the sake of comparison we also provided the $p_{c}-\varepsilon$ curve obtained for compression axis located along [111] direction. It is seen that the Young modulus for [111] direction is greater than those obtained for [001] one.

cubic $(\mathrm{Si}(\mathrm{I}))$ to the high-pressure $\beta$-tin $(\mathrm{Si}(\mathrm{II}))$ phase $[5-$ $8,15]$. In contrast to the case of GaAs, loading of the indenter does not produce the pop-in associated with the mentioned phase change. Instead of that, the release of contact load exhibits so called the pop-out phenomenon being a signature of transformation from $\beta$-tin phase formed during loading to mixture of metastable $\mathrm{Si}$ (III) and $\mathrm{Si}(\mathrm{XII})$ phases. Pop-outs occur as a discontinuous displacement decrease during unloading.

Simulated by us dislocation driven plasticity of the Si nanosphere and an influence of the phase transformations on the plastic nanodeformation of bulk $\mathrm{Si}$ show that the change of mechanism of silicon plasticity is associated with a deconfinement of deformed nanovolume from bulk to nanosphere state. This phenomenon was confirmed by our earlier in situ nanomechanical experiments [21] performed on Si nanospheres with radii ranging from 19 to $169 \mathrm{~nm}$, produced by the Hypersonic Plasma Particle Deposition technique. The load $(P)$ indenter displacement $(h)$ curves recorded for the compressed material revealed two systematic patterns (Fig. 3a). The smaller nanospheres always displayed the characteristic pop-in singularity during loading, while the larger ones a more complex response combining pop-in and popout singularity during loading and unloading, respec- 


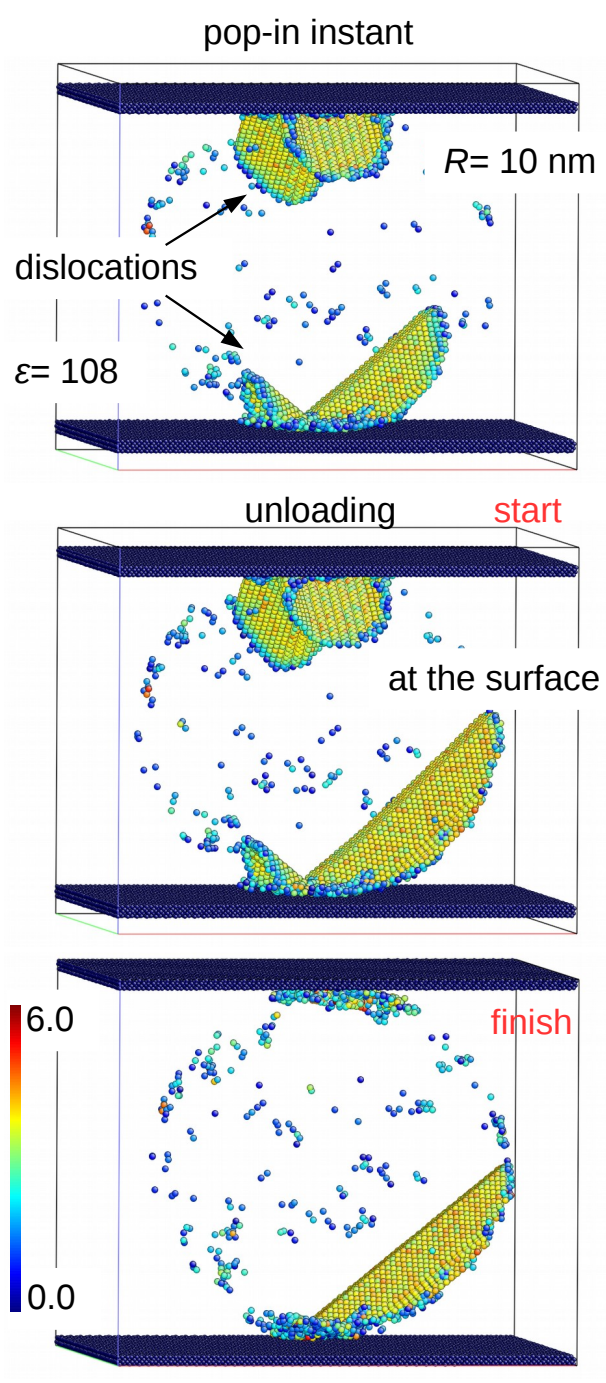

Fig. 2. The atomistic details of dislocation activity in compressed Si nanospheres revealed by means of slipvector [13]. The plastic deformation starts at strain $\varepsilon=0.108$ with nucleation of pairs of perfect dislocations. Unloading performed right after the pop-in leads to irreversible and reversible dislocations' behavior.

tively. Indeed, the pop-in event occurs in nanospheres radii smaller than $67 \mathrm{~nm}$, while the remaining particles $(67 \mathrm{~nm} \leq R \leq 169 \mathrm{~nm}$ ) display combined pop-in/popout effect. The appearance of pop-out proves existence of $\mathrm{Si}(\mathrm{II})$ in stressed bulk crystal or nanosphere, while our MD simulations proved the pop-in phenomenon to be driven by dislocations nucleation in strained silicon nanosphere.

In light of the results we have presented, we propose the concept of deconfinement to reflect a transition from bulk to nanosphere behavior; a process in which deformation is driven by a distinctive set of mechanisms, resulting in an alteration of the very properties of silicon (Fig. 3b). Deconfinement leads to the shift from phase-transformation-dominated incipient plasticity to a dislocation-driven one, exemplified by the pop-
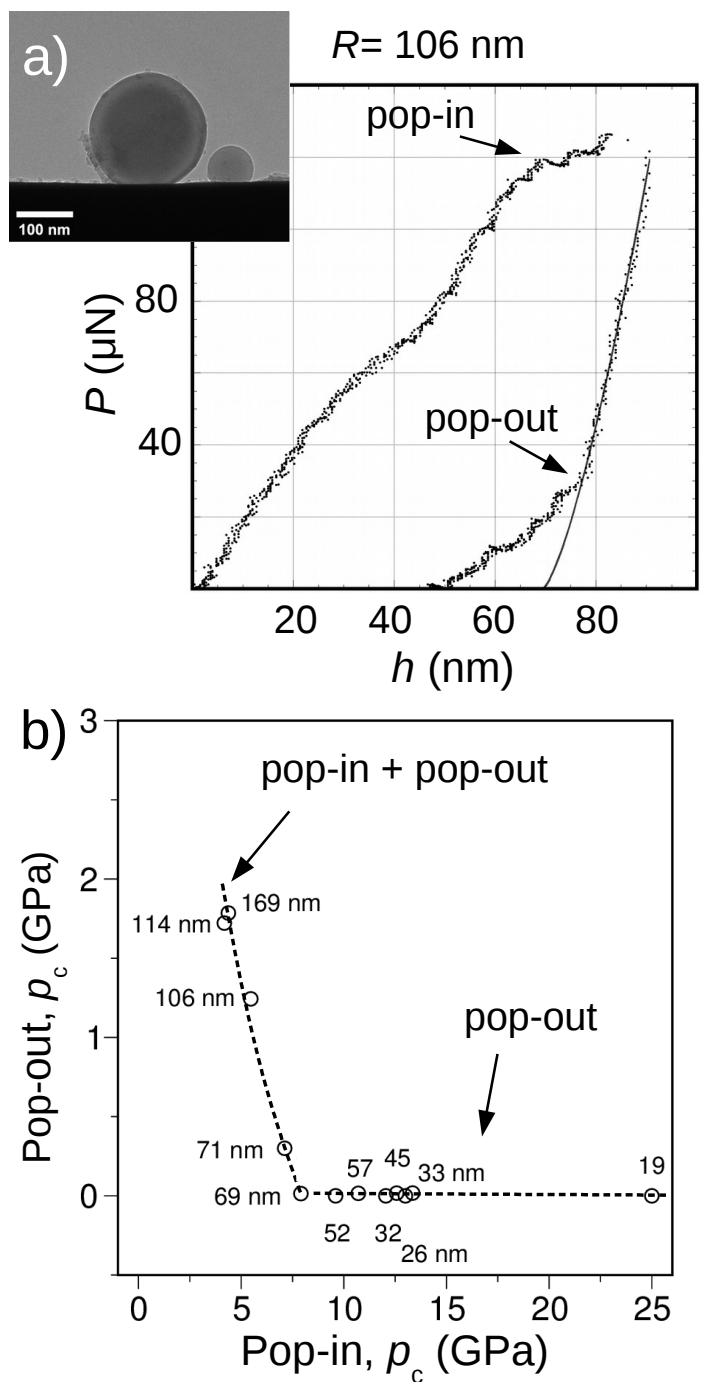

Fig. 3. Results of compression experiment performed for Si-nanosphere with radius of $106 \mathrm{~nm}$ (a). The popin and pop-out events occur for particles whose radius exceeds $67 \mathrm{~nm}$ (b). The plasticity of smaller Si nanoparticles is exclusively dislocation-governed which means that the only pop-in event was observed on the $P-h$ curves.

out $\rightarrow$ pop-out/pop-in $\rightarrow$ pop-in sequence (bulk $\rightarrow$ large nanosphere $\rightarrow$ small nanosphere).

In order to resolve physical foundations of the deconfinement phenomenon, we performed supplementary MD-simulations to model the mechanical response of the bulk Si crystal. The silicon structure was deformed under stress that has been induced by the contact between Si-crystal surface and spherical rigid indenter (radius of $10 \mathrm{~nm}$ ). This allowed us to compare the development of stress distributions in compressed nanosphere $(R=10 \mathrm{~nm})$ as contrasting to this induced by nanoindentation in the bulk Si-crystal. The results provide the detailed information on the hydrostatic stress and von Mises shear stress, respectively. The calculated stress distributions are in agreement with the Hertz contact the- 


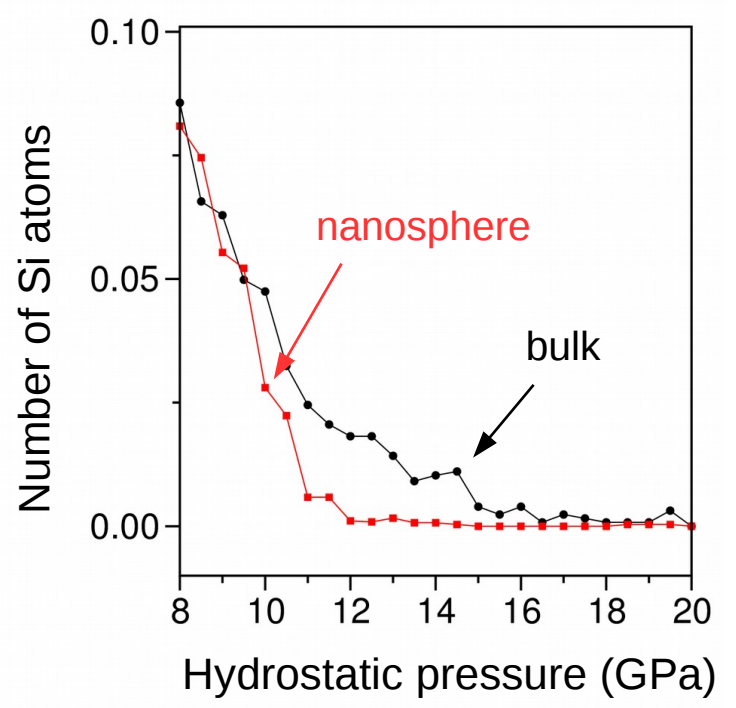

Fig. 4. Number of atoms in the bulk Si subjected to hydrostatic stress higher than $10 \mathrm{GPa}$ is higher than corresponding atoms in $\mathrm{Si}$ nanosphere.

ory. Indeed, we detected the maximum hydrostatic stress right at the contact surface, while the maximum shear stress is located deeper in the investigated Si-crystal.

All of this prompts us to contend that despite of similar straining of bulk Si crystal and Si nanoparticle, the stress distribution within confined (bulk) and deconfined (particle) nanovolume differ each other. Certainly, the maximum value of the shear stress increases from $8.4 \pm 0.6 \mathrm{GPa}$ to $9.6 \pm 0.4 \mathrm{GPa}$ while the maximum value of the hydrostatic pressure decreases. The number of Si atoms that sustain pressure exceeding $10 \mathrm{GPa}$ is significantly higher in the bulk-Si than in silicon nanoparticle (Fig. 4). This brings us to conclusion that the nanoscale Si deconfinement from bulk to nanoparticle is associated with the shear stresses increase accompanied by hydrostatic stress decrease and favors the dislocation-based plasticity in expense of phase transformation scenario.

\section{Conclusions}

In sum, the discovery of the deconfinement effect governing a transition in mechanical response from bulk to nanoparticle behavior is of practical importance, as it paves the way to manipulate the number and activity of dislocations in Si nanoparticles. Given the impact of dislocations on the functional properties of Si nanovolumes, a controlled evolution of dislocation structures in Si nanospheres opens a new possibilities in the development of tunable Si nanodevices with optoelectronic and biomedical applications, where photoluminescence plays a crucial role. The nanoscale deconfinement will benefit processing future nanostructures for electronic, magnetic and optical devices as well as biomedical applications including drug delivery and biosensors as indicated by Cross [22].

\section{Acknowledgments}

This work was supported by the Academy of Finland (research project PROPER). It also received support from National Science Center of Poland under Grant No. DEC-2012/05/B/ST-8/02945. The authors also gratefully acknowledge the CSC-IT Center for Science (Finland) for computation resources.

\section{References}

[1] R. Nowak, F. Yoshida, D. Chrobak, K.J. Kurzydłowki, T. Takagi, T. Sasaki, in: Encyclopedia of Nanoscience and Nanotechnology, Ed. S.H. Nalwa, American Scientific, 2011, p. 313.

[2] C.A. Schuh, J.K. Mason, A.C. Lund, Nature Mater. 4, 617 (2005).

[3] D. Chrobak, K. Nordlund, R. Nowak, Phys. Rev. Lett. 98, 045502 (2007).

[4] R. Nowak, D. Chrobak, S. Nagao, D. Vodnick, M. Berg, A. Tukianen, M. Pessa, Nature Nanotechnol. 4, 287 (2009).

[5] I. Zarudi, J. Zou, L.C. Zhang, Appl. Phys. Lett. 82, 874 (2003).

[6] V. Domnich, Y. Gogotsi, S. Dub, Appl. Phys. Lett. 76, 2214 (2000).

[7] E.T. Lilleodden, J.A. Zimmerman, S.M. Foiles, W.D. Nix, J. Mech. Phys. Solids 51, 901 (2003).

[8] D. Ge, A.M. Minor, E.A. Stach, J.W. Morris, Philos. Mag. 86, 4069 (2006).

[9] N. O'Farrel, A. Houlton, B.R. Horrocks, 1, Int. J. Nanomed. 1, 451 (2006).

[10] W.W. Gerberich, W.M. Mook, C.R. Perrey, C.B. Carter, M.I. Baskes, R. Mukherjee, A. Gidwani, J. Heberlein, P.H. McMurry, S.L. Girshick, J. Mech. Phys. Solids 51, 979 (2003).

[11] F.H. Stillinger, T.A. Weber, Phys. Rev. B 31, 5262 (1985).

[12] J. Godet, L. Pizzagalli, S. Brochard, P. Beauchamp, Phys. Rev. B 70, 054109 (2004).

[13] J.A. Zimmerman, L.C. Kelchner, P.A. Klein, J.C. Hamilton, S.M. Foiles, Phys. Rev. Lett. 87, 165507 (2001).

[14] H. Hertz, J. Reine Angew. Math. 92, 156 (1882).

[15] P. Valentini, W.W. Gerberich, T. Dumitrica, Phys. Rev. Lett. 99, 175701 (2007).

[16] Y. Mo, K.T. Turner, I. Szlufarska, Nature 457, 1116 (2009).

[17] W.W. Gerberich, J.C. Nelson, E.T. Lilleodden, P. Anderson, T.J. Wyrobek, Acta Mater. 44, 3585 (1996).

[18] J. Li, K.J. Van Vliet, T. Zhu, S. Yip, S. Suresh, Nature 418, 307 (2002).

[19] J.K. Mason, A.C. Lund, C.A. Schuh, Phys. Rev. B 73, 054102 (2006).

[20] O.L. Warren, S.A. Downs, T.J. Wyrobek, Z. Metallkd. 95, 287 (2004).

[21] D. Chrobak, N. Tymiak, A. Beaber, O. Ugurlu, W.W. Gerberich, R. Nowak, Nature Nanotechnol. 6, 480 (2011).

[22] G.L.W. Cross, Nature Nanotechnol. 6, 467 (2011). 